\title{
Editorials
}

\section{Understanding the needs of carers of people with psychosis in primary care}

\section{INTRODUCTION}

A couple you have known for years consult with you; they are in despair. Their son's new psychiatric team has just advised them that, as he is now an adult, he will be able to make his own decisions without his parents attending routine appointments. They have watched their son's prospects for a normal life disintegrate over the last 2 years rarely leaving his room, losing his friends, and spending most days without structure or purpose.

Many individuals diagnosed with psychotic conditions live with, or remain in close contact with, informal caregivers, typically close relatives such as their parents, partners, siblings, and children. As in the scenario above, the experience of a family member developing psychosis leaves family members bewildered and frightened, working through high levels of distress and often unsupported or even excluded by healthcare providers. Moreover, this often heralds a long-term commitment by families to support and advocate for an individual while feeling unsupported. As 'family practitioners' GPs are in a unique position within the NHS to engage with and support both the individual with psychosis and their family carers.

Psychotic disorders, such as schizophrenia, are severe and disabling mental health conditions that affect several million people worldwide and rank among the top global contributors to years lived with disability. The onset typically emerges in adolescence and young adulthood, and often during a period when a young person is establishing their independence. The hallmark symptoms include hallucinations and delusions, often persecutory, that can be focused on other people, including relatives and carers. These will often occur alongside marked disturbances in affect, cognition, and social functioning.

In the UK, the pooled incidence rates for psychotic disorders are estimated at 32 per 100000 people with significantly higher rates for black and minority ethnic groups. Annual prevalence rates are estimated at 4 cases per 1000 people, which are mainly attributed to schizophrenia.' Thus, a typical GP can expect to encounter family members involved with about one new presentation of psychosis every other year and provide ongoing support to between 5-10 patients experiencing a severe and enduring mental illness, rates

"In the UK alone, informal carers are estimated to save the public purse approximately 11.25 billion per annum in unpaid care... Improving the health and capacity of informal carers to care more effectively should be a cornerstone of high-quality general practice.

varying with ethnicity and social deprivation.

The deleterious effects of psychosis on an individual's global functioning, social networks, and quality of life are well documented. Evidence confirms that reports of trauma, substance misuse, suicide, and stigma are typically elevated in people with psychosis alongside high levels of unemployment and premature mortality. ${ }^{2}$

To date, pharmacological interventions continue to represent the main treatment approach used in psychosis, although psychological therapies such as cognitive behavioural therapy have an increasing evidence base. However, the nature and complexity of the conditions mean many sufferers, despite optimal treatments and best practice, will experience enduring symptoms and difficulties that impact on social functioning and relationships. ${ }^{2}$

\section{CHALLENGES FACED BY CARERS}

Many individuals diagnosed with psychosis live with, or remain in close contact with, informal carers. Research evidence suggests that patients with support from informal carers can achieve better outcomes, which include fewer inpatient admissions and relapses, better engagement with services and prescribed treatments, and improved mortality rates. Carers play a central role in organising the relevant care and support for patients at initial onset, and in responding to incipient signs of relapse and unmet need. ${ }^{3}$

In the UK alone, informal carers are estimated to save the public purse approximately $€ 1.25$ billion per annum in unpaid care. ${ }^{2}$ The World Health Organization estimated that the burden to individual families from psychotic disorders such as schizophrenia is only exceeded by dementia and quadriplegia. ${ }^{4}$ As part of their caregiving role, many carers may have to deal with confusing and distressing illness symptoms from their relative (for example, persecutory delusions, poor self-care, risky health behaviours, and isolation) alongside a range of difficult behaviours that may, at times, include acts of aggression and self-harm that are stigmatising. ${ }^{3}$ In addition, providing information and facilitating carer support can help and encourage the development of positive relationships, which is important because difficult and negative caregiving relationships are predictive of patient and carer outcomes. This can place a burden on the carer, and risk guilt at a time of relapse. ${ }^{3}$

Although caregiving can be rewarding for some, and associated with positive experiences, there is evidence that many carers report being negatively impacted by their role. Data from general population psychiatric morbidity surveys confirm higher levels of common mental disorders in carer populations, irrespective of disorder, and with an observable deterioration in those providing $>10$ hours of care per week. ${ }^{5}$

Among carers of people with psychosis, approximately 40\% report poor psychological functioning that includes depression and other stress-related disorders. Carer distress can persist over the illness course while also peaking during the early illness phase and inpatient admissions. Reports of burnout and exhaustion have been recorded in carers, including at first episode. Feelings of loss and grief are also highly prevalent in carers of people with psychosis, and not dissimilar in content and presentation to reactions observed following a bereavement. ${ }^{3}$ Comparisons between carers of people with psychosis with non-caregiving peers confirm a poorer quality of life and greater social isolation in the psychosis group. Recently published evidence indicates they are up to 10 times more isolated than non-carers but are also significantly more socially isolated than carers of people with other health conditions. ${ }^{6-8}$ Poor levels of physical health, including sleep difficulties, 
are also found in psychosis carers. Perlick et al ${ }^{8}$ reviewed 264 carers of people with severe mental illness that comprised schizophrenia spectrum conditions; twothirds reported having at least one medical condition in the preceding 5 years, such as arthritis, hypertension, and emphysema, and the remaining one-third reported having at least two medical conditions.

Given their substantial and valued contribution to optimising patient outcomes and the negative impact of caregiving on their own health status, offering support to family carers in psychosis has been included in several treatment publications over the last decade (for example, National Institute for Health and Care Excellence guidance on prevention and management of psychosis and schizophrenia in adults). ${ }^{9}$ However, carers of people with psychosis can feel that their needs are overlooked and neglected by health professionals and services. Carers have been consistent in their requests for opportunities to access trusted information on psychosis and individually tailored support that enables them to cope with their relative's illness and manage the impact on their lives and relationships. The debate continues of how best to meet these needs.

\section{WHAT CAN PRIMARY CARE DO?}

Service reconfiguration and new models of working have ensured that increasing numbers of people with psychosis are being discharged to and cared for at primary care level. Effectively addressing the health needs of patients and improving their outcomes also requires recognition of the varied and distinct needs of their informal carers and consideration of strategies to optimise their outcomes. Effective communication between primary and specialist mental health services is crucial for the patient and their carers; GPs have a role in facilitating communication between the patient and carers and the community mental health team.

Given the illness chronicity, carers of people with psychosis should be considered a vulnerable patient group, and strategies to reduce health deficits should be prioritised, as would be considered for any vulnerable group at higher risk for potentially preventable morbidity.

Recent findings from a national survey of 195364 carers in England highlighted their less positive patient experience when compared with non-carers. This was particularly evident for subgroups of carers such as males and those who were younger..$^{10}$ Such findings are of note in the context of broader research that suggests more than three-quarters of carers identify their GPs as having the greatest influence on being able to improve their lives. ${ }^{11}$ Encouragingly, early pilot data from carer awareness training programmes embedded in primary care settings showed that two-thirds of participating GPs and allied health practitioners reported the training had led to attitudinal changes towards caregivers and a greater confidence in offering support. However, the absence of incentives, enhanced services, training, or other additional resources left some participants doubting whether significant changes for carers could be implemented meaningfully. ${ }^{12}$

In our scenario the parents were under enormous strain and had approached their GP in desperation. Legitimising their understandable concerns with an empathetic approach, based on awareness of the impact of psychosis on the wider family, is an essential first step. We also know that these carers face a future where, despite disproportionately higher levels of physical and mental health needs, they may not access their GPs more than noncaregiver groups. ${ }^{13}$ This health inequality is one that primary care can address through some simple actions: tag the case record to alert opportunistic discussion of carers' health needs; encourage continuity with the same GP; proactively encourage attendance for regular review; and signpost and facilitate access to carer-focused services and resources (for example, www.rethink. org; www.futurelearn.com/courses/caringpsychosis-schizophrenia).

A more systematic, proactive approach might avoid unplanned attendance at both primary care and the emergency department, while also underpinning a more constructive alliance between GPs and carers to better support the individual with psychosis. Improving the health and capacity of informal carers to care more effectively should be a cornerstone of highquality general practice.

\section{Juliana Onwumere,}

Lecturer in Clinical Psychology, King's College London; Honorary Consultant Clinical Psychologist, South London \& Maudsley NHS Foundation Trust.

\section{David Shiers,}

Honorary Research Fellow, University of Manchester, and Carer.

\section{Carolyn Chew-Graham,}

Professor of General Practice Research, Research Institute, Primary Care and Health Sciences, Keele University; Honorary Professor of Primary Care, Primary Care Mental Health, South Staffs and Shropshire Foundation Trust.

\section{ADDRESS FOR CORRESPONDENCE}

Carolyn Chew-Graham

Research Institute, Primary Care and Health

Sciences, Keele University, Keele, Staffordshire ST5 5BG, UK.

\section{E-mail: c.a.chew-grahamakeele.ac.uk}

\section{Provenance}

Commissioned; externally peer reviewed.

\section{DOI: 10.3399/bjgp16X686209}

\section{REFERENCES}

1. Kirkbride JB, Errazuriz A, Croudace TJ, et al. Systematic review of the incidence and prevalence of schizophrenia and other psychoses in England. Department of Health Policy Programme, 2012.

2. Schizophrenia Commission. The abandoned illness: a report from the Schizophrenia Commission. London: Rethink Mental Illness, 2012.

3. Kuipers E, Onwumere J, Bebbington P. Cognitive model of caregiving in psychosis. $\mathrm{Br} \mathrm{J}$ Psychiatry 2010; DOI:10.1192/bjp.bp.109.070466.

4. World Health Organization. Mental health: new understanding, new hope. Geneva: WHO, 2001.

5. Smith L, Onwumere J, Craig T, et al. Mental and physical illness in caregivers: results from an English national survey sample 2007. Br J Psychiatry 2014; 205(3): 197-203.

6. Hayes L, Hawthorne G, Farhall J, et al. Quality of life and social isolation among caregivers of adults with schizophrenia: policy and outcomes. Community Ment Health J 2015; DOI: 10.1007/ s10597-015-9848-6.

7. Magliano L, Fiorillo A, De Rosa C, et al. Family burden in long term diseases: a comparative study in schizophrenia vs. physical disorders. Soc Sci Med 2005; 61(2): 313-322.

8. Perlick DA, Hohenstein JM, Clarkin JF, et al. Use of mental health and primary care services by caregivers of patients with bipolar disorder: a preliminary study. Bipolar Disord 2005; 7(2): 126-135.

9. National Institute for Health and Care Excellence. Psychosis and schizophrenia in adults: prevention and management. CG178. London: NICE, 2014. https://uww.nice.org.uk/ guidance/cg178 (accessed 4 Jul 2016).

10. Thomas GPA, Saunders CL, Roland MO, Paddison CAM. Informal carers' health-related quality of life and patient experience in primary care: evidence from 195,364 carers in England responding to a national survey. BMC Fam Pract 2015; 16: 62.

11. Henwood M. Ignored and invisible? Carers experience of the NHS. London: Carers' National Association, 1998.

12. Jones R, Mackenzie A, Greenwood N, et al. Evaluation of the pilot GP training programme on carers. Kingston University \& St George’s University of London, 2010. http://eprints. kingston.ac.uk/16429/ (accessed 4 Jul 2016).

13. McCrone P, Szmukler G, Kuipers E. Service use and cost associated with caring for people with serious mental illness. J Mental Health 2005; 14(1): $37-47$. 